\title{
Alvocidib Hydrochloride
}

National Cancer Institute

\section{Source}

National Cancer Institute. Alvocidib Hydrochloride. NCI Thesaurus. Code C1571.

A synthetic N-methylpiperidinyl chlorophenyl flavone compound. As an inhibitor of cyclindependent kinase, alvocidib induces cell cycle arrest by preventing phosphorylation of cyclin-dependent kinases (CDKs) and by down-regulating cyclin D1 and D3 expression, resulting in $\mathrm{G} 1$ cell cycle arrest and apoptosis. This agent is also a competitive inhibitor of adenosine triphosphate activity. 